\title{
Prevalence and Risk Factors Associated With Sars-cov-2 Infection in Healthcare Personnel of the Spanish Central Military Hospital. Results of a Case-control Study
}

MARIA JULIA AJEJAS BAZAN ( $\square$ majejas@ucm.es)

Universidad Complutense de Madrid https://orcid.org/0000-0001-5343-3377

CARLOS FUENTES MORA

Hospital Central de la Defensa Gómez Ulla: Hospital Central de la Defensa Gomez Ulla

LUCÍA ELENA BALLESTER ORCAL

Hospital Central de la Defensa Gómez Ulla: Hospital Central de la Defensa Gomez Ulla

JULIA WÄRNBERG

Universidad de Malaga Facultad de Ciencias de la Salud

LUIS HERRERO PÉREZ

Gobierno de España Ministerio de Defensa: Gobierno de Espana Ministerio de Defensa

\section{MIGUEL PUERRO VICENTE}

Universidad de Alcalá de Henares Facultad de Medicina y Ciencias de la Salud: Universidad de Alcala de Henares Facultad de Medicina y Ciencias de la Salud

NAPOLEÓN PÉREZ FARINÓS

Universidad de Málaga Facultad de Medicina: Universidad de Malaga Facultad de Medicina

\section{Research}

Keywords: Coronavirus Infections, Personnel Hospital, Military Personnel, Risk Factors, Transmission

Posted Date: May 7th, 2021

DOl: https://doi.org/10.21203/rs.3.rs-496471/v1

License: (9) This work is licensed under a Creative Commons Attribution 4.0 International License. Read Full License 


\section{Abstract}

Background. Spain is the country with the highest number of health care workers affected by COVID-19 disease in the world. The aim of this study was to describe the epidemiology of SARS-COV-2 infection in HCW at the Gómez Ulla Military Hospital.

Methods. A case-control study was conducted during the first outbreak of COVID 19 at GUMH. The study was extended to the total number of HCW in the hospital who met the inclusion criteria. HCW of the GUMH were civilian and military personnel that included Nursing and Medicine students of Spanish Military Academy, Medicine specialty residents and Nursing residents of Medical Surgical Specialty in Operations (EMQO). An ad hoc questionnaire of 33 items was made up. The questionnaire was sent by e-mail to the entire population of study. With this questionnaire personnel were classified into sick (cases) or healthy (controls).

Results and Conclusions. 150 professionals answered the questionnaire. Cases were defined as those who tested positive in the diagnostic tests $(n=28,20.7 \%)$ and controls were those who tested negative $(n=107,79.3 \%)$. Therefore, the prevalence of SARSCoV-2 in the GUMH was $20.7 \%$. Of the total number of cases, $64.3 \%$ were men ( $p<0.05)$, with a mean age of 47.1 (SD 13.3), with a mean BMI of 25.3 (SD 3.8), 48.2 \% were overweighted. Of the total cases, $59.3 \%$ had A blood group type and $69.2 \%$ were Rh positive. $50 \%$ were physicians, $32.1 \%$ were nurses and $17.9 \%$ were auxiliary nurses $(p<0.05)$. Cases and controls with vitamin $D$ deficiency and who took supplements had a lower risk of suffering COVID-19, with significant differences. Fever, cough and diarrhea were found in at least $50 \%$ of the sample with significant differences.

\section{Introduction}

The pandemic caused by SARS-Cov-2 (COVID-19) first identified in Wuhan, China, remains as a global health problem. Following the experience during the 2002 outbreak of severe acute respiratory syndrome (SARS), an important factor in controlling transmission is the protection of healthcare workers (HCW), as 1725 cases among HCW were infected with SARS (1). In the ongoing epidemic, infection among HCW has been increasing since the first 15 affected cases were reported in Wuhan (2). It is necessary to know the risk factors and nosocomial transmission to protect the health of staff.

Spain is the country with the highest number of HCW affected by COVID-19 disease in the world. A report by the European Centre for Disease Control and Prevention (ECDC) highlights that 20\% of COVID-19 in Spain affects this group, while in Italy this percentage is 10\%, and in the United States and China 3\% and 3.8\%, respectively (3). Up to May 21st, 2020, a total of 250287 cases of COVID-19 were reported to the National Epidemiological Surveillance Network (RENAVE) in Spain. 40921 cases correspond to HCW (4).

It is necessary to adopt primary preventive measures to reduce morbidity and mortality among HCW. To establish such measures, it is important to know which factors are linked to the different health determinants of the most exposed armed forces HCW. To know these causes will contribute to the design, development, and implementation of public health measures to respond effectively to the ongoing SARS-CoV-2 epidemic.

The aim of this study was to describe the epidemiology of SARS-COV-2 infection in HCW at the Gómez Ulla Military Hospital (GUMH, Madrid, Spain). Secondary aims were to describe the prevalence of COVID-19 infection in healthcare personnel at baseline, to analyze the probability of exposure to different risk factors in cases versus controls, to detail the signs and symptoms presented by cases, and to assess which factors influenced the onset of signs and symptoms.

\section{Methods}

\section{Design, setting and participants}

A case-control study was conducted during the first outbreak of COVID 19 at GUMH. The total study population were 1171 health professionals working in that hospital. No sampling was selected, as the study was extended to the total number of HCW in the hospital who met the inclusion criteria. HCW of the GUMH were civilian and military personnel that included Nursing and Medicine students of Spanish Military Academy, Medicine specialty residents and Nursing residents of Medical Surgical Specialty in Operations (EMQO). 
All HCW who were not physicians, nurses, Medicine or Nursing residents, or Nursing assistants, were excluded from the study.

\section{Study variables}

The collected variables collected were age, sex, height, weight; body Mass Index (BMI) was calculated as weight $(\mathrm{kg}) / \mathrm{height}^{2}\left(\mathrm{~m}^{2}\right)$; blood type ( $\mathrm{ABO}$ and $\mathrm{Rh}$ ); type of healthcare professional; physician or nurse specialty if they had; civilian or military personnel; years of experience, (coded as $<10$ years, $10-20$ years and $>20$ years); field of work (hospital emergency, emergency areas enabled for this purpose, intensive care, hospitalization, other); to receive seasonal influenza vaccine in 2019-2020 (yes, no); to suffer high blood pressure (HBP), diabetes, heart disease, Chronic Obstructive Pulmonary Disease (COPD), asthma, malignant tumors or other chronical disease; tobacco consumption (yes, no, ex-smoker); alcohol consumption (never drank, less than once a week, drink at least once a week); measurement of stress and distress in professionals through the Perceived Stress Scale (PSS), in its validated Spanish version, reduced 4-item version. In addition, the following variables were collected in relation to COVID-19 infection: currently working (yes, no); COVID-19 signs and symptoms currently present (yes, no); to have been tested for COVID-19 (yes, no); type of COVID-19 test (PCR, rapid antigen test, ELISA); test result (positive, negative); to be daily exposed to COVID-19 patients (yes, little exposure, no exposure); average time of exposure to COVID patients, per shift ( $<5$ hours, $5-8$ hours, $>5$ hours); work shifts performed (morning shift, afternoon shift, night shift, 24-hour shifts, 12-hour shifts, back-up); number of hours of daily work (8, 10, $12,14,16,24)$; days off weekly in the period of COVID-19 care $(0,1,2$, more than 2); extent to which you have received adequate training in handling personal protective equipment (PPE) (a lot, a lot, a little, not at all); PPE removal training (always, often, occasionally, rarely, hardly ever, never); to keep the removal steps safely (always, often, occasionally, hardly ever, never); whenever left a more contaminated room or area, to remove the outermost layer of PPE (always, often, occasionally, rarely, almost never, never); amount of protective material (a lot, quite enough, little, none); adequate material (yes, no); to re-use the material (yes, no); if re-used material, to indicate which (gloves, mask, impervious gown, tights, hat, protective screen, goggles).

An ad hoc questionnaire of 33 items was made up. The questionnaire was sent by e-mail to the entire population of study. With this questionnaire personnel were classified into sick (cases) or healthy (controls).

\section{Ethical aspects}

All individuals received an initial section in the questionnaire in which they were informed in detail of the details, objectives, and other aspects of the study. Likewise, this project was evaluated and approved by the Research Ethics Committee of the Góme Ulla Military Hospital. All data were treated according to the Organic Law 3/2018, of December 5, on Data Protection and Guarantee of Digital Rights (LOPDGDD) and subsequent amendments, as well as the Declaration of Helsinki of the World Medical Association, on ethical principles for medical research on human beings and finally the Law $41 / 2002$, of November 14 , basic regulating patient autonomy and rights and obligations regarding information and clinical documentation.

\section{Statistical analysis}

All risk factors were assessed and the probability of having been exposed or not to these factors was calculated versus the controls.

Variables were described with absolute and relative frequencies. To assess which quantitative variables followed a normal distribution, the Kolmogorov-Smirnof test was performed. To assess whether there was a statistically significant association $(p<$ 0.05) between the case-control status and of the study variables, Pearson Chi-Squared Test (qualitative variables) and Student's $t$ test (quantitative variables) were used. To assess the independent association of the factors studied with the disease, odds ratios (OR) with their $95 \%$ confidence intervals were calculated using logistic regression models. The models were adjusted for the confounding variables that were considered appropriate, and the existence of interaction between them was evaluated. Statistical processing of the data was carried out using SPSS (Statistical Package for Social Sciences) V25.0 for Windows.

\section{Results}

Of the 1171 health professionals of the initial population, 703 (62.0\%) were women, had a mean age of 48.7 years, 577 (49.3\%) were nurses, $365(31.2 \%)$ physicians and 229 (19.5\%) nursing assistants. 
150 professionals answered the questionnaire. $56.2 \%$ were women, with a mean age of 46.7 years (SD 13.4). $47.6 \%$ were nurses, $29.6 \%$ were physicians and $27.3 \%$ were auxiliary nurses. $63.2 \%$ had 10 years of experience and $66.4 \%$ were military personnel. $36.7 \%$ worked in intensive care, $35.9 \%$ in hospitalization service and $32.0 \%$ in the emergency service. $62.5 \%$ were aged 50 years or over. Mean BMI was $24.9 \mathrm{~kg} / \mathrm{m}^{2}$ (SD 3.9) and $61.7 \%$ were normal weighted. $47.7 \%$ had A blood group and $77.3 \%$ were Rh positive. The results presented in this study refer to the personnel who answered to the questionnaire (Table 1). 
Table 1

Sample characteristics, prevalence and factors associated with COVID-19.

\begin{tabular}{|c|c|c|c|c|c|c|c|}
\hline & $\begin{array}{l}\text { Total n } \\
(\%)\end{array}$ & $\begin{array}{l}\text { Cases n } \\
(\%)\end{array}$ & IC $95 \%$ & $\begin{array}{l}\text { Controls n } \\
(\%)\end{array}$ & IC $95 \%$ & p-value & OR (IC $95 \%)$ \\
\hline \multicolumn{6}{|l|}{$\operatorname{Sex}(n=132)$} & \multirow[t]{3}{*}{$0.024^{*}$} & \multirow{3}{*}{$\begin{array}{l}3.2(1.1- \\
8.6)^{\star \star \star}\end{array}$} \\
\hline Man & $\begin{array}{l}60 \\
(45.5)\end{array}$ & $18(64.3)$ & $\begin{array}{l}(56.6- \\
72.0)\end{array}$ & $42(40.4)$ & $\begin{array}{l}(32.5- \\
48.3)\end{array}$ & & \\
\hline Woman & $\begin{array}{l}72 \\
(54.5)\end{array}$ & $10(35.7)$ & $\begin{array}{l}(28.0- \\
43.4)\end{array}$ & $62(59.6)$ & $\begin{array}{l}(51.1- \\
67.5)\end{array}$ & & \\
\hline \multicolumn{8}{|l|}{ Age $(n=150)$} \\
\hline $\mathrm{M}(\mathrm{DT})$ & $\begin{array}{l}46.7 \\
(13.4)\end{array}$ & $\begin{array}{l}47.1 \\
(13.3)\end{array}$ & $\begin{array}{l}(7.9- \\
18.9)\end{array}$ & $44.1(14.4)$ & $\begin{array}{l}(8.8- \\
20.0)\end{array}$ & $0.332 * \star$ & $\begin{array}{l}1.0(1.0- \\
1.1) \star \star \star\end{array}$ \\
\hline \multicolumn{8}{|l|}{ Age intervals $(n=128)$} \\
\hline$<=50$ years & $\begin{array}{l}80 \\
(62.5)\end{array}$ & $14(50.0)$ & $\begin{array}{l}(42.0- \\
58.8)\end{array}$ & $66(66.0)$ & $\begin{array}{l}(58.4- \\
73.6)\end{array}$ & \multirow[t]{2}{*}{$0.122 *$} & \\
\hline$>50$ years & $\begin{array}{l}48 \\
(37.5)\end{array}$ & $14(50.0)$ & $\begin{array}{l}(42.0- \\
58.8)\end{array}$ & $34(34.0)$ & $\begin{array}{l}(26.4- \\
41.6)\end{array}$ & & \\
\hline \multicolumn{8}{|l|}{ IMC } \\
\hline $\mathrm{M}(\mathrm{DT})$ & $\begin{array}{l}24.9 \\
(3.9)\end{array}$ & $\begin{array}{l}25.3 \\
(3.8)\end{array}$ & $(0.7-6.9)$ & $24.2(3.7)$ & $(0.7-6.7)$ & \multicolumn{2}{|l|}{$0.181^{\star *}$} \\
\hline \multicolumn{8}{|c|}{$\begin{array}{l}\text { Weight interpretation of the IMC } \\
(n=130)\end{array}$} \\
\hline Normopeso & $\begin{array}{l}79 \\
(60.8)\end{array}$ & $14(51.8)$ & $\begin{array}{l}(43.2- \\
60.4)\end{array}$ & $65(63.1)$ & $\begin{array}{l}(54.8- \\
71.4)\end{array}$ & \multirow[t]{2}{*}{$0.286^{\star}$} & \\
\hline $\begin{array}{l}\text { Excess weight } \\
\text { (Everweight/Obesity) }\end{array}$ & $\begin{array}{l}51 \\
(39.2)\end{array}$ & $13(48.2)$ & $\begin{array}{l}(39.6- \\
56.8)\end{array}$ & $38(36.9)$ & $\begin{array}{l}(28.6- \\
45.2)\end{array}$ & & \\
\hline \multicolumn{8}{|l|}{ Blood Group ( $n=126)$} \\
\hline 0 & $\begin{array}{l}39 \\
(30.9)\end{array}$ & $7(25.9)$ & $\begin{array}{l}(18.9- \\
32.9)\end{array}$ & $32(32.3)$ & $\begin{array}{l}(24.8- \\
39.8)\end{array}$ & \multirow[t]{4}{*}{$0.129 *$} & \\
\hline A & $\begin{array}{l}61 \\
(48.4)\end{array}$ & $16(59.3)$ & $\begin{array}{l}(51.4- \\
67.2)\end{array}$ & $45(45.5)$ & $\begin{array}{l}(37.5- \\
53.5)\end{array}$ & & \\
\hline$B$ & $\begin{array}{l}18 \\
(14.3)\end{array}$ & $4(14.8)$ & $\begin{array}{l}(9.1- \\
20.5)\end{array}$ & $14(14.1)$ & $\begin{array}{l}(8.5- \\
19.7)\end{array}$ & & \\
\hline$A B$ & $8(6.4)$ & $0(0.0)$ & $(0.0-0.0)$ & $8(8.1)$ & $\begin{array}{l}(3.7- \\
12.5)\end{array}$ & & \\
\hline \multicolumn{8}{|l|}{$\mathrm{RH}(\mathrm{n}=124)$} \\
\hline Positivo & $\begin{array}{l}99 \\
(79.8)\end{array}$ & $18(69.2)$ & $\begin{array}{l}(61.8- \\
76.6)\end{array}$ & $81(82.7)$ & $\begin{array}{l}(76.6- \\
88.8)\end{array}$ & \multirow[t]{2}{*}{$0.169 *$} & \\
\hline Negativo & $\begin{array}{l}25 \\
(20.2)\end{array}$ & $8(30.8)$ & $\begin{array}{l}(23.4- \\
38.2)\end{array}$ & $17(17.3)$ & $\begin{array}{l}(11.2- \\
23.4)\end{array}$ & & \\
\hline \multicolumn{8}{|c|}{ Health professionals $(n=134)$} \\
\hline Physician & $\begin{array}{l}38 \\
(28.3)\end{array}$ & $14(50.0)$ & $\begin{array}{l}(42.0- \\
58.0)\end{array}$ & $24(22.6)$ & $\begin{array}{l}(15.9- \\
29.3)\end{array}$ & $0.017 *$ & $\begin{array}{l}5.8(0.9- \\
34.7) \star \star \star\end{array}$ \\
\hline \multicolumn{8}{|c|}{ Source: Own elaboration; } \\
\hline
\end{tabular}




\begin{tabular}{|c|c|c|c|c|c|c|c|}
\hline & $\begin{array}{l}\text { Total n } \\
\text { (\%) }\end{array}$ & $\begin{array}{l}\text { Cases n } \\
(\%)\end{array}$ & IC $95 \%$ & $\begin{array}{l}\text { Controls n } \\
(\%)\end{array}$ & IC $95 \%$ & p-value & OR (IC $95 \%)$ \\
\hline Nurse & $\begin{array}{l}61 \\
(45.5)\end{array}$ & $9(32.1)$ & $\begin{array}{l}(24.6- \\
39.6)\end{array}$ & $52(49.1)$ & $\begin{array}{l}(41.1- \\
57.1)\end{array}$ & & \\
\hline Auxiliary nurse & $\begin{array}{l}35 \\
(26.2)\end{array}$ & $5(17.9)$ & $\begin{array}{l}(11.8- \\
24.0)\end{array}$ & $30(28.3)$ & $\begin{array}{l}(20.8- \\
35.2)\end{array}$ & & \\
\hline \multicolumn{8}{|c|}{ Years of experience $(n=120)$} \\
\hline$<10$ years & $\begin{array}{l}39 \\
(32.5)\end{array}$ & $6(22.2)$ & $\begin{array}{l}(15.5- \\
28.9)\end{array}$ & $33(35.5)$ & $\begin{array}{l}(27.8- \\
43.2)\end{array}$ & \multirow{2}{*}{\multicolumn{2}{|c|}{$0.422^{\star}$}} \\
\hline$>=10$ years & $\begin{array}{l}81 \\
(67.5)\end{array}$ & $21(77.8)$ & $\begin{array}{l}(71.1- \\
84.5)\end{array}$ & $60(64.5)$ & $\begin{array}{l}(56.8- \\
72.2)\end{array}$ & & \\
\hline \multicolumn{8}{|c|}{ Type of Administration $(n=133)$} \\
\hline Military & $\begin{array}{l}85 \\
(63.9)\end{array}$ & $20(74.1)$ & $\begin{array}{l}(67.1- \\
81.1)\end{array}$ & $65(61.3)$ & $\begin{array}{l}(53.5- \\
69.1)\end{array}$ & \multirow{2}{*}{\multicolumn{2}{|c|}{$0.218^{\star}$}} \\
\hline Civil & $\begin{array}{l}48 \\
(36.1)\end{array}$ & $7(25.9)$ & $\begin{array}{l}(18.9- \\
32.9)\end{array}$ & $41(38.7)$ & $\begin{array}{l}(30.9- \\
46.5)\end{array}$ & & \\
\hline \multicolumn{8}{|c|}{ Work Environment ( $n=123)$} \\
\hline Intensive Care & $\begin{array}{l}47 \\
(38.2)\end{array}$ & $9(34.6)$ & $\begin{array}{l}(27.0- \\
42.2)\end{array}$ & 38 (39.2) & $\begin{array}{l}(31.4- \\
47.0)\end{array}$ & \multirow{3}{*}{\multicolumn{2}{|c|}{$0.384^{\star}$}} \\
\hline Hospitalization & $\begin{array}{l}46 \\
(37.4)\end{array}$ & $8(30.8)$ & $\begin{array}{l}(23.4- \\
38.2)\end{array}$ & $38(39.2)$ & $\begin{array}{l}(31.4- \\
47.0)\end{array}$ & & \\
\hline Urgency & $\begin{array}{l}30 \\
(24.4)\end{array}$ & $9(34.6)$ & $\begin{array}{l}(27.7- \\
42.2^{-}\end{array}$ & $21(21.6)$ & $\begin{array}{l}(15.0- \\
28.2)\end{array}$ & & \\
\hline \multicolumn{8}{|c|}{ Flu vaccine $(n=131)$} \\
\hline Yes & $\begin{array}{l}28 \\
(21.4)\end{array}$ & $6(21.4)$ & $\begin{array}{l}(14.4- \\
28.4)\end{array}$ & $22(21.3)$ & $\begin{array}{l}(14.3- \\
28.3)\end{array}$ & \multirow{2}{*}{\multicolumn{2}{|c|}{$0.994^{\star}$}} \\
\hline No & $\begin{array}{l}103 \\
(78.6)\end{array}$ & $22(78.6)$ & $\begin{array}{l}(72.0- \\
85.2)\end{array}$ & $81(78.7)$ & $\begin{array}{l}(72.0- \\
85.2)\end{array}$ & & \\
\hline \multicolumn{8}{|c|}{$\begin{array}{l}\text { Suffering from chronic disease ( } n \\
=130 \text { ) }\end{array}$} \\
\hline Yes & $\begin{array}{l}31 \\
(24.2)\end{array}$ & $7(25.9)$ & $\begin{array}{l}(18.9- \\
32.9)\end{array}$ & $24(23.3)$ & $\begin{array}{l}(16.5- \\
30.1)\end{array}$ & \multirow{2}{*}{\multicolumn{2}{|c|}{$0.776^{\star}$}} \\
\hline No & $\begin{array}{l}99 \\
(77.3)\end{array}$ & $20(74.1)$ & $\begin{array}{l}(67.1- \\
81.1^{-}\end{array}$ & 79 (76.7) & $\begin{array}{l}(69.9- \\
83.5)\end{array}$ & & \\
\hline \multicolumn{8}{|c|}{ Physical Activity $(n=130)$} \\
\hline Yes & $\begin{array}{l}105 \\
(80.8)\end{array}$ & $21(80.7)$ & $\begin{array}{l}(73.9- \\
87.5)\end{array}$ & $84(80.7)$ & $\begin{array}{l}(73.9- \\
87.5)\end{array}$ & \multirow{2}{*}{\multicolumn{2}{|c|}{$0.981^{*}$}} \\
\hline No & 25(19.2) & $5(19.2)$ & $\begin{array}{l}(12.4- \\
26.0)\end{array}$ & $20(19.2)$ & $\begin{array}{l}(12.4- \\
26.0)\end{array}$ & & \\
\hline \multicolumn{8}{|c|}{ Vitamin D Deficiency $(n=97)$} \\
\hline Yes & $\begin{array}{l}18 \\
(18.5)\end{array}$ & $6(27.2)$ & $\begin{array}{l}(18.3- \\
36.1)\end{array}$ & $12(11.2)$ & $\begin{array}{l}(4.9- \\
17.5)\end{array}$ & \multirow{2}{*}{\multicolumn{2}{|c|}{$0.018^{*}$}} \\
\hline \multicolumn{6}{|c|}{ Source: Own elaboration; } & & \\
\hline $\begin{array}{l}\text { * Chi-cuadrado, } \\
\text { absolute frequer }\end{array}$ & $\begin{array}{l}0.05, \mathrm{M} \text { - } \\
\text { dex. }\end{array}$ & thmetic m & n, DT- sta & rd deviation & S/NC- dol & now/no a & swer, n- \\
\hline
\end{tabular}




\begin{tabular}{|c|c|c|c|c|c|c|c|}
\hline & $\begin{array}{l}\text { Total } \mathbf{n} \\
(\%)\end{array}$ & $\begin{array}{l}\text { Cases n } \\
(\%)\end{array}$ & IC $95 \%$ & $\begin{array}{l}\text { Controls n } \\
(\%)\end{array}$ & IC $95 \%$ & p-value & OR (IC $95 \%)$ \\
\hline No & $\begin{array}{l}79 \\
(81.5)\end{array}$ & $16(72.8)$ & $\begin{array}{l}(63.9- \\
81.7)\end{array}$ & $63(58.9)$ & $\begin{array}{l}(49.1- \\
68.7)\end{array}$ & & \\
\hline \multicolumn{8}{|c|}{$\begin{array}{l}\text { Vitamin D supplementation }(n= \\
133)\end{array}$} \\
\hline Yes & $\begin{array}{l}16 \\
(12.0)\end{array}$ & $7(25.0)$ & $\begin{array}{l}(18.1- \\
31.9)\end{array}$ & $9(8.6)$ & $\begin{array}{l}(4.1- \\
13.1)\end{array}$ & \multirow{2}{*}{\multicolumn{2}{|c|}{$0.047 *$}} \\
\hline No & $\begin{array}{l}117 \\
(88.0)\end{array}$ & $21(75.0)$ & $\begin{array}{l}(68.1- \\
81.9)\end{array}$ & $96(91.4)$ & $\begin{array}{l}(86.9- \\
95.9)\end{array}$ & & \\
\hline \multicolumn{8}{|l|}{ Smoking ( $n=134)$} \\
\hline Yes & $\begin{array}{l}17 \\
(12.7)\end{array}$ & $2(7.4)$ & $\begin{array}{l}(3.2- \\
11.6)\end{array}$ & $15(14.0)$ & $\begin{array}{l}(8.4- \\
19.6)\end{array}$ & \multirow{3}{*}{\multicolumn{2}{|c|}{$0.559 *$}} \\
\hline No & $\begin{array}{l}84 \\
(62.7)\end{array}$ & $19(70.4)$ & $\begin{array}{l}(63.3- \\
77.1)\end{array}$ & $65(60.7)$ & $\begin{array}{l}(52.9- \\
68.5)\end{array}$ & & \\
\hline Ex-smoker & $\begin{array}{l}33 \\
(24.6)\end{array}$ & $6(22.2)$ & $\begin{array}{l}(15.5- \\
28.9)\end{array}$ & $27(25.3)$ & $\begin{array}{l}(18.3- \\
32.1)\end{array}$ & & \\
\hline \multicolumn{8}{|l|}{ Alcohol $(n=133)$} \\
\hline Less than once a week & $\begin{array}{l}82 \\
(61.6)\end{array}$ & $15(53.6)$ & $\begin{array}{l}(45.6- \\
61.6)\end{array}$ & $67(63.8)$ & $\begin{array}{l}(56.1- \\
71.5)\end{array}$ & \multirow{2}{*}{\multicolumn{2}{|c|}{$0.322^{\star}$}} \\
\hline Drink at least once a week & $\begin{array}{l}51 \\
(38.4)\end{array}$ & $13(46.4)$ & $\begin{array}{l}(38.4- \\
54.4)\end{array}$ & $38(36.2)$ & $\begin{array}{l}(28.5- \\
43.9)\end{array}$ & & \\
\hline \multicolumn{8}{|l|}{ Stress Scale } \\
\hline M (DT) & $\begin{array}{l}3.47 \\
(1.9)\end{array}$ & $\begin{array}{l}2.86 \\
(2.1)\end{array}$ & $(0.0-4.4)$ & $3.92(1.9)$ & $(0.0-4.1)$ & $0.012^{\star \star}$ & \\
\hline \multicolumn{8}{|l|}{ Stress Type $(n=134)$} \\
\hline Low & $\begin{array}{l}109 \\
(81.3)\end{array}$ & $25(89.3)$ & $\begin{array}{l}(84.4- \\
94.2)\end{array}$ & $84(79.2)$ & $\begin{array}{l}(72.7- \\
85.7)\end{array}$ & \multirow{2}{*}{\multicolumn{2}{|c|}{$0.225^{\star}$}} \\
\hline Medium & $\begin{array}{l}25 \\
(18.7)\end{array}$ & $3(10.7)$ & $\begin{array}{l}(5.8- \\
15.6)\end{array}$ & $22(20.8)$ & $\begin{array}{l}(14.3- \\
27.3)\end{array}$ & & \\
\hline \multicolumn{8}{|l|}{ Source: Own elaboration; } \\
\hline
\end{tabular}

Of the 150 professionals who answered the questionnaire, 15 did not undergo any diagnostic test, so they could not be classified as cases or controls. Cases were defined as those who tested positive in the diagnostic tests $(n=28,20.7 \%)$ and controls were those who tested negative $(n=107,79.3 \%)$. Therefore, the prevalence of SARS-CoV-2 in the GUMH was $20.7 \%$. Of the total number of cases, $64.3 \%$ were men $(p<0.05)$, with a mean age of 47.1 (SD 13.3), with a mean BMI of 25.3 (SD 3.8), $48.2 \%$ were overweighted. Of the total cases, $59.3 \%$ had A blood group type and $69.2 \%$ were Rh positive. $50 \%$ were physicians, $32.1 \%$ were nurses and 17.9 $\%$ were auxiliary nurses $(p<0.05)$. Likewise, $77.8 \%$ of the cases reported having 10 or more years of experience and $74.1 \%$ were military personnel. Concerning the work environment, the percentage of cases was similar between those who worked in intensive care (34.6\%), hospitalization (30.8\%) or emergency (34.6\%). In $78.6 \%$ of the cases no flu vaccine was administered. $74.1 \%$ did not present any type of chronic disease, $72.8 \%$ did not present vitamin D deficiency $(p<0.05)$ and $75.0 \%$ did not take vitamin D supplements $(p<0.05)$. Regarding healthy habits, $70.4 \%$ reported not smoking and $53.6 \%$ consumed alcohol less than once a week. In addition, the mean stress among the cases was $2.86(2.1)(p<0.05)$ and $10.7 \%$ reported feeling a medium level of stress (Table 1). 
Concerning the factors of direct exposure to COVID-19, which could have influenced transmission, $82.1 \%$ of the cases reported having been exposed to COVID-19 patients, $64.3 \%$ were exposed for at least five hours or more, $77.8 \%$ of the cases worked during the morning shift $(p<0.05)$, with an average daily working hours of 8.6 (SD 1.0) and a mean number of days free of work of 1.25 (SD 0.7). The percentage of cases that reported having done a lot of training for handling PPE was $3.7 \%$ compared to $32.1 \%$ of cases that reported not having done any training. There were no cases among those who reported having always been supervised in the removal of PPE versus $57.7 \%$ of the cases who reported having never been supervised. The $25.0 \%$ of the cases reported having safely followed the steps for PPE removal compared to $33.3 \%$ who did not, finding a statistically significant association ( $p$ $=0.009$ ). $52.2 \%$ of the cases did not always remove the outermost layer when leaving contaminated areas, while $69.8 \%$ of the controls always did so. The percentage of cases and controls that reported that there was little protective material was $81.5 \%$ in cases and $77.6 \%$ controls. $60.7 \%$ of the cases and $68.3 \%$ of the controls rated the material they had as adequate, and $78.6 \%$ of the cases and $90.5 \%$ of the controls reported that they had re-used some element of the protective material sometimes (Table 2). 
Table 2

Epidemiological background of exposure.

\begin{tabular}{|c|c|c|c|c|c|c|c|}
\hline & $\begin{array}{l}\text { Total } \\
(n= \\
150)\end{array}$ & $\begin{array}{l}\text { Cases } \\
\text { n (\%) }\end{array}$ & $\begin{array}{l}\operatorname{IC} 95 \\
\%\end{array}$ & $\begin{array}{l}\text { Controls } \\
\mathrm{n}(\%)\end{array}$ & $\begin{array}{l}\text { IC } 95 \\
\%\end{array}$ & $\begin{array}{l}\mathrm{p}- \\
\text { value }\end{array}$ & $\begin{array}{l}\text { OR (IC } \\
95 \%)\end{array}$ \\
\hline \multicolumn{8}{|c|}{$\begin{array}{l}\text { Exposed to COVID-19 patients in their daily work ( } n= \\
\text { 134) }\end{array}$} \\
\hline Yes & $\begin{array}{l}107 \\
(79.9)\end{array}$ & $\begin{array}{l}23 \\
(82.1)\end{array}$ & $\begin{array}{l}(75.6- \\
88.6)\end{array}$ & $\begin{array}{l}84 \\
(79.2)\end{array}$ & $\begin{array}{l}(72.3- \\
86.1)\end{array}$ & \multirow[t]{3}{*}{0.499} & \\
\hline Little exposure & $\begin{array}{l}22 \\
(16.4)\end{array}$ & $\begin{array}{l}5 \\
(17.9)\end{array}$ & $\begin{array}{l}(11.4- \\
24.4)\end{array}$ & $\begin{array}{l}17 \\
(16.0)\end{array}$ & $\begin{array}{l}(9.8- \\
22.2)\end{array}$ & & \\
\hline No exposure & $\begin{array}{l}5 \\
(3.7)\end{array}$ & $0(0.0)$ & $\begin{array}{l}(0.0- \\
0.0)\end{array}$ & $5(4.7)$ & $\begin{array}{l}(1.1- \\
8.3)\end{array}$ & & \\
\hline \multicolumn{8}{|c|}{ Average exposure time $(n=131)$} \\
\hline$<5$ hours & $\begin{array}{l}37 \\
(28.2)\end{array}$ & $\begin{array}{l}10 \\
(35.7)\end{array}$ & $\begin{array}{l}(27.6- \\
43.8)\end{array}$ & $\begin{array}{l}27 \\
(26.2)\end{array}$ & $\begin{array}{l}(18.7- \\
33.7)\end{array}$ & \multirow[t]{2}{*}{0.323} & \\
\hline$>=5$ hours & $\begin{array}{l}94 \\
(71.8)\end{array}$ & $\begin{array}{l}18 \\
(64.3)\end{array}$ & $\begin{array}{l}(56.1- \\
72.5)\end{array}$ & $\begin{array}{l}76 \\
(73.8)\end{array}$ & $\begin{array}{l}(66.3- \\
81.3)\end{array}$ & & \\
\hline \multicolumn{8}{|c|}{ Working hours $(n=131)$} \\
\hline Morning shift & $\begin{array}{l}77 \\
(58.8)\end{array}$ & $\begin{array}{l}21 \\
(77.8)\end{array}$ & $\begin{array}{l}(70.7- \\
84.9)\end{array}$ & $\begin{array}{l}56 \\
(53.8)\end{array}$ & $\begin{array}{l}(45.3- \\
62.3)\end{array}$ & \multirow[t]{2}{*}{$0.024^{*}$} & \multirow{2}{*}{$\begin{array}{l}1.7 \\
(0.5- \\
5.7)\end{array}$} \\
\hline Rest of shifts & $\begin{array}{l}54 \\
(41.2)\end{array}$ & $\begin{array}{l}6 \\
(22.2)\end{array}$ & $\begin{array}{l}(15.1- \\
29.3)\end{array}$ & $\begin{array}{l}48 \\
(46.2)\end{array}$ & $\begin{array}{l}(37.7- \\
54.7)\end{array}$ & & \\
\hline \multicolumn{8}{|c|}{ Number of working hours per day $(n=134)$} \\
\hline M (DT) & $\begin{array}{l}8.7 \\
(1.7)\end{array}$ & $\begin{array}{l}8.6 \\
(1.0)\end{array}$ & $\begin{array}{l}(3.9- \\
13.3)\end{array}$ & $8.8(1.9)$ & $\begin{array}{l}(4.0- \\
13.6)\end{array}$ & 0.673 & \\
\hline \multicolumn{8}{|c|}{ Weekly days off $(n=134)$} \\
\hline M (DT) & $\begin{array}{l}1.32 \\
(0.7)\end{array}$ & $\begin{array}{l}1.25 \\
(0.7)\end{array}$ & $\begin{array}{l}(0.0- \\
2.1)\end{array}$ & $\begin{array}{l}1.44 \\
(0.7)\end{array}$ & $\begin{array}{l}(0.0- \\
2.1)\end{array}$ & 0.251 & \\
\hline \multicolumn{8}{|c|}{ Adequate training for handling PPE $(n=129)$} \\
\hline A lot & $\begin{array}{l}8 \\
(6.3)\end{array}$ & $1(3.7)$ & $\begin{array}{l}(0.5- \\
6.9)\end{array}$ & $7(6.9)$ & $\begin{array}{l}(2.6- \\
11.2)\end{array}$ & \multirow[t]{4}{*}{0.077} & \\
\hline Quite a lot & $\begin{array}{l}35 \\
(27.1)\end{array}$ & $\begin{array}{l}9 \\
(32.1)\end{array}$ & $\begin{array}{l}(24.2- \\
40.0)\end{array}$ & $\begin{array}{l}26 \\
(25.7)\end{array}$ & $\begin{array}{l}\left(18.3^{-}\right. \\
33.1\end{array}$ & & \\
\hline A little & $\begin{array}{l}63 \\
(48.8)\end{array}$ & $\begin{array}{l}9 \\
(32.1)\end{array}$ & $\begin{array}{l}(24.2- \\
40.0)\end{array}$ & $\begin{array}{l}54 \\
(53.5)\end{array}$ & $\begin{array}{l}(45.1- \\
61.9)\end{array}$ & & \\
\hline Not at all & $\begin{array}{l}23 \\
(17.8)\end{array}$ & $\begin{array}{l}9 \\
(32.1)\end{array}$ & $\begin{array}{l}(24.2- \\
40.0)\end{array}$ & $\begin{array}{l}14 \\
(13.9)\end{array}$ & $\begin{array}{l}(8.0- \\
19.8)\end{array}$ & & \\
\hline \multicolumn{8}{|c|}{$\begin{array}{l}\text { In the removal of the PPE, it has always been under } \\
\text { supervision. }(n=126)\end{array}$} \\
\hline Always & $\begin{array}{l}6 \\
(4.9)\end{array}$ & $0(0.0)$ & $\begin{array}{l}(0.0- \\
0.0)\end{array}$ & $6(6.0)$ & $\begin{array}{l}(2.0- \\
10.0)\end{array}$ & \multirow[t]{3}{*}{0.562} & \\
\hline Often & $\begin{array}{l}9 \\
(7.1)\end{array}$ & $2(7.7)$ & $\begin{array}{l}(3.2- \\
12.2)\end{array}$ & $7(7.0)$ & $\begin{array}{l}(2.7- \\
11.3)\end{array}$ & & \\
\hline Ocassionally & $\begin{array}{l}12 \\
(9.5)\end{array}$ & $2(7.7)$ & $\begin{array}{l}(3.2- \\
12.2)\end{array}$ & $\begin{array}{l}10 \\
(10.0)\end{array}$ & $\begin{array}{l}(4.9- \\
15.1)\end{array}$ & & \\
\hline
\end{tabular}




\begin{tabular}{|c|c|c|c|c|c|c|c|}
\hline & $\begin{array}{l}\text { Total } \\
(\mathrm{n}= \\
150)\end{array}$ & $\begin{array}{l}\text { Cases } \\
\mathrm{n}(\%)\end{array}$ & $\begin{array}{l}\text { IC } 95 \\
\%\end{array}$ & $\begin{array}{l}\text { Controls } \\
\mathrm{n}(\%)\end{array}$ & $\begin{array}{l}\text { IC } 95 \\
\%\end{array}$ & $\begin{array}{l}\mathrm{p}- \\
\text { value }\end{array}$ & $\begin{array}{l}\text { OR (IC } \\
95 \%)\end{array}$ \\
\hline Hardly ever & $\begin{array}{l}41 \\
(32.5)\end{array}$ & $\begin{array}{l}7 \\
(26.9)\end{array}$ & $\begin{array}{l}(19.4- \\
34.4)\end{array}$ & $\begin{array}{l}34 \\
(34.0)\end{array}$ & $\begin{array}{l}(26.0- \\
42.0)\end{array}$ & & \\
\hline Never & $\begin{array}{l}58 \\
(46.0)\end{array}$ & $\begin{array}{l}15 \\
(57.7)\end{array}$ & $\begin{array}{l}(49.3- \\
66.1)\end{array}$ & $\begin{array}{l}43 \\
(43.0)\end{array}$ & $\begin{array}{l}(34.6- \\
51.4)\end{array}$ & & \\
\hline \multicolumn{8}{|c|}{ You have safely followed withdrawal steps $(n=124)$} \\
\hline Always & $\begin{array}{l}53 \\
(42.7)\end{array}$ & $\begin{array}{l}6 \\
(25.0)\end{array}$ & $\begin{array}{l}(17.7- \\
32.3)\end{array}$ & $\begin{array}{l}47 \\
(47.0)\end{array}$ & $\begin{array}{l}(38.5- \\
55.5)\end{array}$ & \multirow[t]{3}{*}{$0.009 *$} & \multirow{3}{*}{$\begin{array}{l}6.4 \\
(1.6- \\
25.8)^{\star}\end{array}$} \\
\hline Often & $\begin{array}{l}53 \\
(42.7)\end{array}$ & $\begin{array}{l}10 \\
(41.7)\end{array}$ & $\begin{array}{l}(33.4- \\
50.0)\end{array}$ & $\begin{array}{l}43 \\
(43.0)\end{array}$ & $\begin{array}{l}(34.6- \\
51.4)\end{array}$ & & \\
\hline Hardly ever & $\begin{array}{l}18 \\
(14.6)\end{array}$ & $\begin{array}{l}8 \\
(33.3)\end{array}$ & $\begin{array}{l}(25.3- \\
41.3)\end{array}$ & $\begin{array}{l}10 \\
(10.0)\end{array}$ & $\begin{array}{l}(4.9- \\
15.1)\end{array}$ & & \\
\hline \multicolumn{8}{|c|}{$\begin{array}{l}\text { When leaving contaminated areas, was the } \\
\text { outermost layer removed? }(n=119)\end{array}$} \\
\hline Always & $\begin{array}{l}78 \\
(65.5)\end{array}$ & $\begin{array}{l}11 \\
(47.8)\end{array}$ & $\begin{array}{l}(39.3- \\
56.3)\end{array}$ & $\begin{array}{l}67 \\
(69.8)\end{array}$ & $\begin{array}{l}(62.0- \\
77.6)\end{array}$ & \multirow[t]{2}{*}{0.46} & \\
\hline Hardly ever & $\begin{array}{l}41 \\
(34.5)\end{array}$ & $\begin{array}{l}12 \\
(52.2)\end{array}$ & $\begin{array}{l}(43.7- \\
60.7)\end{array}$ & $\begin{array}{l}29 \\
(30.2)\end{array}$ & $\begin{array}{l}(22.4- \\
38.0)\end{array}$ & & \\
\hline \multicolumn{8}{|c|}{ In relation to the material, there have been $(n=134)$} \\
\hline Enough & $\begin{array}{l}29 \\
(21.6)\end{array}$ & $\begin{array}{l}5 \\
(18.5)\end{array}$ & $\begin{array}{l}(11.9- \\
25.1)\end{array}$ & $\begin{array}{l}24 \\
(22.4)\end{array}$ & $\begin{array}{l}(15.3- \\
29.5)\end{array}$ & \multirow[t]{2}{*}{0.659} & \\
\hline Little & $\begin{array}{l}105 \\
(78.4)\end{array}$ & $\begin{array}{l}22 \\
(81.5)\end{array}$ & $\begin{array}{l}(74.9- \\
88.1)\end{array}$ & $\begin{array}{l}83 \\
(77.6)\end{array}$ & $\begin{array}{l}(70.5- \\
84.7)\end{array}$ & & \\
\hline \multicolumn{8}{|c|}{ Suitable material $(n=132)$} \\
\hline Yes & $\begin{array}{l}88 \\
(66.7)\end{array}$ & $\begin{array}{l}17 \\
(60.7)\end{array}$ & $\begin{array}{l}(52.4- \\
69.0)\end{array}$ & $\begin{array}{l}71 \\
(68.3)\end{array}$ & $\begin{array}{l}(60.4- \\
76.2)\end{array}$ & \multirow[t]{2}{*}{0.452} & \\
\hline No & $\begin{array}{l}44 \\
(33.3)\end{array}$ & $\begin{array}{l}11 \\
(39.3)\end{array}$ & $\begin{array}{l}(31.0- \\
47.6)\end{array}$ & $\begin{array}{l}33 \\
(31.7)\end{array}$ & $\begin{array}{l}(23.8- \\
39.6)\end{array}$ & & \\
\hline \multicolumn{8}{|c|}{ Have you reused the material? $(n=133)$} \\
\hline Yes & $\begin{array}{l}117 \\
(88.0)\end{array}$ & $\begin{array}{l}22 \\
(78.6)\end{array}$ & $\begin{array}{l}(71.7- \\
85.5)\end{array}$ & $95(90.5$ & $\begin{array}{l}(85.5- \\
95.5)\end{array}$ & \multirow[t]{2}{*}{0.204} & \\
\hline No & $\begin{array}{l}16 \\
(12.0)\end{array}$ & $\begin{array}{l}6 \\
(21.4)\end{array}$ & $\begin{array}{l}(14.5- \\
28.3)\end{array}$ & $10(9.5)$ & $\begin{array}{l}(4.5- \\
14.5)\end{array}$ & & \\
\hline
\end{tabular}

Concerning the clinical presentation, $60.7 \%$ of the cases presented fever, $50.0 \%$ cough and diarrhea and $46.4 \%$ headache. Only 14.3 $\%$ of the cases presented pneumonia and respiratory distress. Significance was found when they presented fever, sore throat, pneumonia, cough, diarrhea and other respiratory signs (Table 3). Differences were also found in relation to fever and sex, being higher in men $(88.2 \%)$ than in women $(11.8 \%)$, in those who presented chronic comorbidities (58.8\%) versus those who did not $(41.2 \%)$, as well as in those who reported not having vitamin D deficiency (57.1\%) versus those who did (42.9 \%). A statistically significant association was found between sore throat and those who did not receive the flu vaccine (54.5\%) vs. those who did $(45.5 \%)(p<0.05)$. Differences were also found between cases with pneumonia and overweight (100.0\%), 0 blood group (50.0\%), and those who did not perform physical activity $(75.0 \%)$. Cough was associated with those cases with 10 years or more of experience $(61.5 \%)$. On the other hand those cases that presented diarrhea were significantly associated with not taking vitamin D 
supplements $(57.1 \%)(p<0.05)$ and cases with respiratory distress were more among those with 0 blood group $(75.0 \%)(p<0.05)$. In addition, women presented other respiratory signs in a higher percentage $(75 \%)$ than men $(25 \%)$, with a significant association (Table 3). 
Table 3

Most frequent signs and symptoms and factors associated with their appearance.

\begin{tabular}{|c|c|c|c|c|c|c|c|c|}
\hline & $\begin{array}{l}\text { Fever }^{a} \\
\mathrm{n}(\%)\end{array}$ & $\begin{array}{l}\text { Sore } \\
\text { throattab } \\
n(\%)\end{array}$ & Pneumoniac & $\begin{array}{l}\text { Cough } \\
n(\%)\end{array}$ & Headachea $^{e}$ & Diarrhea $^{f}$ & $\begin{array}{l}\text { Respiratory } \\
\text { distress }^{9}\end{array}$ & $\begin{array}{l}\text { Other } \\
\text { respiratory } \\
\text { signs }^{\text {h }}\end{array}$ \\
\hline Total $^{a, b, c, d, f, f, h}$ & $\begin{array}{l}17 \\
(60.7)\end{array}$ & $\begin{array}{l}11 \\
(39.3)\end{array}$ & $4(14.3)$ & $\begin{array}{l}14 \\
(50.0)\end{array}$ & $13(46.4)$ & $14(50.0)$ & $4(14.3)$ & $4(14.3)$ \\
\hline IC $95 \%$ & $\begin{array}{l}(52.5- \\
68.9)\end{array}$ & $\begin{array}{l}(31.1- \\
47.5)\end{array}$ & $(8.4-20.2)$ & $\begin{array}{l}(41.6- \\
58.4)\end{array}$ & $(38.0-54.8)$ & $\begin{array}{l}(41.6- \\
58.4)\end{array}$ & $(8.4-20.2)$ & $(8.4-20.2)$ \\
\hline \multicolumn{9}{|l|}{ Sex ${ }^{a, h}$} \\
\hline Man & $\begin{array}{l}15 \\
(88.2)\end{array}$ & $9(81.8)$ & $4(100.0)$ & $\begin{array}{l}11 \\
(78.6)\end{array}$ & $7(53.8)$ & $10(71.4)$ & $4.0(100.0)$ & $1(25.0)$ \\
\hline Woman & $\begin{array}{l}2 \\
(11.8)\end{array}$ & $2(18.2)$ & $0(0.0)$ & $\begin{array}{l}3 \\
(21.4)\end{array}$ & $6(46.2)$ & $4(28.6)$ & $0.0(0.0)$ & $3(75.0)$ \\
\hline \multicolumn{9}{|l|}{$\begin{array}{l}\text { BMI Weight } \\
\text { interpretation c }\end{array}$} \\
\hline Normopeso & $\begin{array}{l}7 \\
(41.2)\end{array}$ & $5(45.5)$ & $0(0.0)$ & $\begin{array}{l}7 \\
(50.0)\end{array}$ & $6(46.2)$ & $7(50.0)$ & $1(25.0)$ & $3(75.0)$ \\
\hline $\begin{array}{l}\text { Exceso de peso } \\
\text { (Sobrpeso/Obesidad) }\end{array}$ & $\begin{array}{l}10 \\
(58.8)\end{array}$ & $6(54.5)$ & $4(100.0)$ & $\begin{array}{l}7 \\
(50.0)\end{array}$ & $7(53.8)$ & $7(50.0)$ & $3(75.0)$ & $1(25.0)$ \\
\hline \multicolumn{9}{|l|}{$\begin{array}{l}\text { Blood group }(n= \\
126)^{\mathrm{c}, \mathrm{g}}\end{array}$} \\
\hline 0 & $\begin{array}{l}5 \\
(31.3)\end{array}$ & $2(20.0)$ & $2(50.0)$ & $\begin{array}{l}3 \\
(23.1)\end{array}$ & $5(38.5)$ & $4(28.6)$ & $3(75.0)$ & $0(0.0)$ \\
\hline A & $\begin{array}{l}9 \\
(56.3)\end{array}$ & $8(80.0)$ & $0(0.0)$ & $\begin{array}{l}8 \\
(61.5)\end{array}$ & $6(46.2)$ & $7(50.0)$ & $0(0.0)$ & $2(66.7)$ \\
\hline B & $\begin{array}{l}2 \\
(12.4)\end{array}$ & $0(0.0)$ & $2(50.0)$ & $\begin{array}{l}2 \\
(15.4)\end{array}$ & $2(15.4)$ & $3(21.4)$ & $1(25.0)$ & $1(33.3)$ \\
\hline$A B$ & $0(0.0)$ & $0(0.0)$ & $0(0.0)$ & $0(0.0)$ & $0(0.0)$ & $0(0.0)$ & $0(0.0)$ & $0(0.0)$ \\
\hline \multicolumn{9}{|l|}{ Years of experience $^{d}$} \\
\hline$<10$ years & $\begin{array}{l}4 \\
(25.0)\end{array}$ & $1(10.0)$ & $0(0.0)$ & $\begin{array}{l}5 \\
(38.5)\end{array}$ & $2(16.7)$ & $2(15.4)$ & $0(0.0)$ & $2(50.0)$ \\
\hline >= 10 years & $\begin{array}{l}12 \\
(75.0)\end{array}$ & $9(90.0)$ & $4(100.0)$ & $\begin{array}{l}8 \\
(61.5)\end{array}$ & $10(83.3)$ & $11(84.6)$ & $4(100.0)$ & $2(50.0)$ \\
\hline \multicolumn{9}{|l|}{ Influenza vaccine $e^{b}$} \\
\hline Yes & $\begin{array}{l}5 \\
(29.4)\end{array}$ & $5(45.5)$ & $1(25.0)$ & $\begin{array}{l}3 \\
(21.4)\end{array}$ & $1(7.7)$ & $2(14.3)$ & $1(25.0)$ & $1(25.0)$ \\
\hline No & $\begin{array}{l}12 \\
(70.6)\end{array}$ & $6(54.5)$ & $3(75.0)$ & $\begin{array}{l}11 \\
(78.6)\end{array}$ & $12(92.3)$ & 12 (85.7) & $3(75.0)$ & $3(75.0)$ \\
\hline \multicolumn{9}{|l|}{$\begin{array}{l}\text { Suffering from } \\
\text { chronic disease }^{a}\end{array}$} \\
\hline Yes & $\begin{array}{l}10 \\
(58.8)\end{array}$ & 8 (72.7) & $1(25.0)$ & $\begin{array}{l}11 \\
(78.6)\end{array}$ & $10(76.9)$ & $9(6.3)$ & $2(50.0)$ & $4(100.0)$ \\
\hline No & $\begin{array}{l}7 \\
(41.2)\end{array}$ & 3 (27.3) & $3(75.0)$ & $\begin{array}{l}3 \\
(21.4)\end{array}$ & $3(23.1)$ & 5 (35.7) & $2(50.0)$ & $0(0.0)$ \\
\hline
\end{tabular}

Source: Own elaboration 


\begin{tabular}{|c|c|c|c|c|c|c|c|c|}
\hline & $\begin{array}{l}\text { Fevera }^{a} \\
\mathrm{n}(\%)\end{array}$ & $\begin{array}{l}\text { Sore } \\
\text { throattab } \\
n(\%)\end{array}$ & Pneumonia ${ }^{c}$ & $\begin{array}{l}\text { Cough } \\
n(\%)\end{array}$ & Headachea ${ }^{e}$ & Diarrhea $^{f}$ & $\begin{array}{l}\text { Respiratory } \\
\text { distress }^{9}\end{array}$ & $\begin{array}{l}\text { Other } \\
\text { respiratory } \\
\text { signsh }^{h}\end{array}$ \\
\hline \multicolumn{9}{|l|}{ Physical activityc } \\
\hline Yes & $\begin{array}{l}13 \\
(81.3)\end{array}$ & 9 (81.8) & $1(25.0)$ & $\begin{array}{l}10 \\
(76.9)\end{array}$ & $8(66.7)$ & $9(69.2)$ & $2(50.0)$ & $3(75.0)$ \\
\hline No & $\begin{array}{l}3 \\
(18.7)\end{array}$ & $2(18.2)$ & $3(75.0)$ & $\begin{array}{l}3 \\
(23.1)\end{array}$ & 4 (33.3) & $4(30.8)$ & $2(50.0)$ & $1(25.0)$ \\
\hline \multicolumn{9}{|l|}{ Vitamin D deficiency ${ }^{a}$} \\
\hline Yes & $\begin{array}{l}6 \\
(42.9)\end{array}$ & $3(37.5)$ & $1(33.3)$ & $\begin{array}{l}2 \\
(20.0)\end{array}$ & $3(30.0)$ & 3 (27.3) & $1(50.0)$ & $0(0.0)$ \\
\hline No & $\begin{array}{l}8 \\
(57.1)\end{array}$ & $5(62.5)$ & $2(66.7)$ & $\begin{array}{l}8 \\
(80.0)\end{array}$ & $7(70.0)$ & $8(72.7)$ & $1(50.0)$ & $2(100.0)$ \\
\hline \multicolumn{9}{|l|}{$\begin{array}{l}\text { Vitamin D } \\
\text { supplementation } f\end{array}$} \\
\hline Yes & $\begin{array}{l}5 \\
(29.4\end{array}$ & $3(27.3)$ & $1(25.0)$ & $\begin{array}{l}2 \\
(14.3)\end{array}$ & $3(23.1)$ & $6(42.9)$ & $1(25.0)$ & $0(0.0)$ \\
\hline No & $\begin{array}{l}12 \\
(70.6)\end{array}$ & $8(72.7)$ & $3(75.0)$ & $\begin{array}{l}12 \\
(85.7)\end{array}$ & $10(76.9)$ & $8(57.1)$ & $3(75.0)$ & $4(100.0)$ \\
\hline \multicolumn{9}{|l|}{ Alcohold } \\
\hline $\begin{array}{l}\text { Less than once a } \\
\text { week }\end{array}$ & $\begin{array}{l}11 \\
(64.7)\end{array}$ & $6(54.5)$ & $3(75.0)$ & $\begin{array}{l}12 \\
(85.7)\end{array}$ & $7(53.8)$ & $8(57.1)$ & $3(75.0)$ & $3(75.0)$ \\
\hline $\begin{array}{l}\text { Drink at least once a } \\
\text { week }\end{array}$ & $\begin{array}{l}6 \\
(35.3)\end{array}$ & $5(45.5)$ & $1(25.0)$ & $\begin{array}{l}2 \\
(14.3)\end{array}$ & $6(46.2)$ & $6(42.9)$ & $1(25.0)$ & $1(25.0)$ \\
\hline
\end{tabular}

\section{Discussion}

The questionnaire was distributed to 1171 individuals of a total of 1260.89 professionals were discarded because they did not meet the inclusion criteria. The response rate was $12.8 \%$, which is lower than in other studies, where the response rate ranged from $86.7 \%$ (only 83 individuals were sent the questionnaire) [5] to 73.8\% [6]. It should be taken into account that of the 150 professionals, 15 did not undergo any diagnostic test and therefore could not be classified as a case or control, so there were 135 classified participants. A sample design with random sampling and stratification by sex and type of health professional should have included 107 women (72 were included) and 66 men (60 were included), 84 nurses (compared to 61 in our study), 53 physicians (compared to 38 in our study) and 34 nursing assistants (35 were included). The COVID 19 prevalence obtained in this Hospital was $20.7 \%$. In other studies the seroprevalence ranged from $0.75 \%$ (95\% $\mathrm{Cl} 0.0-8.13)$ [6], to $16.4 \%$ in a study done in Spain [7] or $18.5 \%$ in a study done in Ontario [8]. A total of $54.5 \%$ of the healthcare personnel who answered to the questionnaire were women, similar figures were found in the controls (59.6\%), but the majority of cases were men (64.3 $\mathrm{Cl} 95 \% 56.1-72.5)$, and a statistically significant association was found. Other studies have found different results. In two of them carried out in Spain on health personnel (2020), $23.4 \%$ and $23.8 \%$ of the cases, respectively, were men [4] and another study in Girona showed a percentage of women of $90.1 \%$ [7]. Another study in Wuhan had $50.0 \%$ of women [5], another in Canada had $81.7 \%$ [8], another in Buenos Aires had a percentage of women of $71.8 \%$ [6] and another in Germany had a percentage was 73.0\%. This difference may have been because many medical officers from the Military Health Corps were commissioned, in reserve status (personnel who cease to be on active duty when they reach 61 years of age). All the personnel were male and with a high age range, which may have increased the number of cases and explained the increase in male cases (41.7\% vs. $28.6 \%)$. The mean BMI $\left(24.9 \mathrm{~kg} / \mathrm{m}^{2}, \mathrm{SD}\right.$ $3.9)$ of the total and controls was within the normal weight range, while the mean BMI of the cases was elevated $\left(25.3 \mathrm{~kg} / \mathrm{m}^{2}, \mathrm{SD}\right.$ 3.8) and was in the overweight range. One study used data from the UK Biobank ( $n=285817)$ to show that being overweight 
increased the risk of COVID-19 by $44.0 \%$ (relative risk [RR] $=1.44 ; 95 \% \mathrm{Cl}, 1.08-1.92 ; p=0.010$ )[10]. Two studies showed that the odds of having COVID-19 were increased by $30 \%(\mathrm{OR}=1.30 ; 95 \% \mathrm{Cl}, 1.09-1.54 ; p=0.003)[11]$ and $38 \%(\mathrm{OR}=1.38 ; p<0.001)[10]$ respectively, among overweight/obese individuals. The mechanisms responsible for the increased prevalence of COVID-19 in overweight/obese individuals are unknown. Knowledge of other viral infections, such as influenza, provide insight into how excess weight increases the risk of COVID-19 severity. Understanding why this pathology increases the risk of severe COVID-19 is critical to ensure appropriate preventive and interventional therapies against this novel coronavirus.

The percentage of cases with $\mathrm{A}+$ blood type was higher than the other groups. The difference was not statiscally significant, but clinically relevant. Similar data were found in a study done in China where group A was associated with a higher risk of COVID-19 infection compared to $A B$ and $O$ types [12]. In another study authors suggest that while $A$ type may play a role in increasing susceptibility to COVID-19 infection, 0 type may be somewhat protective. However, when infected, blood type does not appear to influence clinical outcome [13]. Another study done in Changsha First Hospital, China, the proportion of patients with A blood type in the COVID-19 group was significantly higher than that in the control group ( $36.9 \%$ vs. $27.5 \%, p=0.006)$ and concluded that patients with A blood type had a higher risk of SARS-CoV-2 infection, while 0 type was associated with a lower risk, indicating that certain ABO blood groups correlated with susceptibility to SARS-CoV-2 [14].

Concerning the professional group physicians were overrepresented in the cases with respect to the controls, with a statistically significant difference, as was found in another study conducted in a Wuhan Hospital [5]. Different results were found in other studies where the professional groups that presented higher susceptibility were nurses and assistants who spent more time in contact with patients where the values ranged from 20.2-52\%[8][7]. This fact could be due to the performance of bronchoscopies, intubations, or site examinations where exposure levels were higher compared to the care activities performed by nurses and auxiliaries, except for the suctioning activities of ICU staff [15]. Cases with 10 or more years of experience and membership in the military administration were proportional to the total and controls.

The percentage of cases who received the influenza vaccine was very low compared to those who did not, but in the same proportion between cases, controls and totals, and no difference was observed. Several studies show that influenza vaccination does not increase the risk of COVID-19 infection [16]. This study provides trust against speculation that influenza vaccine increases the risk of COVID-19 infection. In addition, other studies claim that influenza vaccination may be associated with lower severity and lower mortality from COVID-19 [17][18].

Concerning vitamin D, those with vitamin D deficiency who also took supplements had a lower risk of COVID-19, in the total and both in the cases and controls, with a significant association. The factor that produces positive effects, reducing transmission and improving prognosis, is vitamin D supplementation and not the deficit itself. Several studies reported an association between CRP positivity and vitamin $D$ deficiency. The exact efficacy of vitamin $D$ supplementation for the prevention or as adjunctive treatment of COVID-19 remains to be determined, but several ongoing randomized controlled trials (RCTs) are actively investigating these potential benefits [19][20].

Low stress was associated to COVID-19 (low stress level in $89.3 \%$ cases vs. medium stress level $10.7 \%$ cases). These results were not consistent with another study that reported that the presence of psychosocial risks had important consequences for the quality of care and increased the probability of errors [21].

Likewise, the number of cases among personnel who reported being more exposed was higher than the rest (82.1\% vs. $0.0 \%)$, and no differences were found, since the number of exposed controls was also very high with respect to those not exposed. This fact could suggest that exposure is not as important as nosocomial or community transmission. The same results were not found in a study done in Spain, where greater direct exposure led to a higher number of infections [7]. It would be advisable to maintain a safe distance between professionals, hand hygiene and the use of personal protective equipment. The number of cases among personnel who worked during the morning shift and who had fewer days off than the controls was higher than in other shifts or in the case of the controls, with a statistically significant difference. The morning shift is the one with the greatest work overload and the highest number of health professionals. These two factors, nosocomial transmission and overload, are two important factors to be taken into account for prevention [24]. It would be advisable to increase the number of healthcare personnel during this shift to reduce overload; other studies have obtained similar data [25]. 
Continuing with the epidemiological risk antecedents, the cases had fewer days off (1.25 SD 0.7) than the controls (1.44 SD 0.7). In relation to protective equipment, there were hardly any cases among those who reported adequate training in the use of the equipment.

Concerning signs and symptoms, fever, cough and diarrhea were found in at least $50 \%$ of the sample with significant differences, and the proportion of cases that also showed other symptoms such as headache or sore throat was not negligible [26][27]. In a study conducted in Spain [4], fever and cough were also the most representative signs. Fever was identified more frequently and with significant differences, in men with chronic diseases and without vitamin D deficiency [4]. Sore throat was identified among those who had not been vaccinated against influenza. In a specific analysis, it was observed that health workers with pneumonia were significantly overweight and did not practice any type of sporting activity compared to those who did not have pneumonia. On the other hand, cough was significantly more frequent in healthcare personnel with more than 10 years of experience.

The most important limitation of this study refers to the sample. This difference could have been due to the short time available to the health personnel or to exhaustion after the practice of health care. Likewise, if a sample size calculation had been made with an error percentage of $5 \%$ and a confidence level of $95 \%$, the number of individuals should have been 173 as opposed to the 150 that made up our study.

\section{Conclusions}

The prevalence of SARS-CoV-2 in the HCDGU HCWS was $20.7 \%$.

$64.3 \%$ of the cases were men ( $p<0.05)$, with a mean age of 47.1 (SD 13.3), the mean BMI of the cases was elevated to the overweight range. In the distribution by professional group in cases with respect to controls and total study participants, physicians were overrepresented, with statistically significant differences. Cases and controls with vitamin D deficiency and who took supplements had a lower risk of suffering COVID-19, with significant differences. The number of cases among morning shift personnel and with fewer days off than controls was higher than in other shifts, with a statistically significant association.

Fever, cough and diarrhea were found in at least $50 \%$ of the sample with significant differences, in addition to headache or sore throat.

\section{Declarations}

\section{Authors' contributions}

MJAB designed this study and obtained funding. CFM, LEBO and LHP analyzed and collected the data. MJAB, MPV, JW and NPF wrote the manuscript. NPF, CFM, MPV and LHE provided critical revisions that are important for the intellectual content. All authors read and approved the final manuscript.

\section{Acknowledgments}

To all the healthcare personnel who have worked at the Hospital Central de la Defensa and who have collaborated in this research study despite their physical and mental exhaustion.

\section{Funding}

Not applicable.

\section{Availability of data and materials}

The datasets used and/or analyzed during the current study are available from the corresponding author upon reasonable request.

\section{Ethics approval and consent to participate}

This trial was approved by the Ethics Committee for Research on Drugs of the "Gómez Ulla" Central Military Defense Hospital (33_20). Informed consent was obtained from all participants.

Page 15/17 


\section{Consent for publication}

Not applicable.

\section{Competing interests}

All authors declare no conflicts of interest.

\section{References}

1. Hsin DHC, Macer DRJ. Heroes of SARS: Professional roles and ethics of health care workers. J Infect. 2004;49(3):210-5.

2. Li X, Xu S, Yu M, Wang K, Tao Y, Zhou Y, et al. Risk factors for severity and mortality in adult COVID-19. J Allergy Clin Immunol. 2020; https://doi.org/10.1016/j.jaci.2020.04.006.

3. ECDC. Coronavirus disease 2019 (COVID-19) in the EU/EEA and the UK, ninth update. Stockholm;

4. RENAVE. CNE.CNM (ISCIII). Informe sobre la situación de COVID-19 en personal sanitario en España a 14 de mayo de 2020. Madrid; 2020.

5. Ran L, Chen X, Wang Y, Wu W, Zhang L, Tan X. Risk Factors of Healthcare Workers With Coronavirus Disease 2019: A Retrospective Cohort Study in a Designated Hospital of Wuhan in China. Clin Infect Dis. 2020;71(16):2218-2221. doi:10.1093/cid/ciaa287

6. Silva A, Aguirre MF, Ballejo C, Marro MJ, Gamarnik A, Vargas G, Pifano M,Varela MT, Garcia E, Lawrynowicz A,Uez O,Pagano I. Seroprevalence of the SARS-CoV-2 infection in health workers of the Sanitary Region VIII, at province of Buenos Aires. medRxiv. doi: https://doi.org/10.1101/2020.09.07.20189050https.://doi.org/10.1101/2020.09.07.20189050

7. Trullàs JC, Vilardell I, Blasco M, J. Herediae J.Rev Clin Esp. 2020 Nov; 220(8): 529-531.

8. Kevin L, Schwartz MD, Achonu C, Buchan SA, Brown KA, Lee B, Whelan M, Wu JC, Garber G. COVID-19 infections among Healthcare Workers and Transmission within Households. medRxiv. doi: https://doi.org/10.1101/2020.06.12.20129619

9. Nienhaus A, HodInt R. J. Environ. Public Health 2020, 17, 4881; doi:10.3390/ijerph1713488

10. Ho FK , Celis - Morales CA , Gray SR, et al. Factores de riesgo modificables y no modificables para COVID-19: resultados del UK Biobank . $2020: 28.04 .202083295$

11. Wu J , Li W, Shi X, et al. El tratamiento antiviral temprano contribuye a aliviar la gravedad y mejorar el pronóstico de los pacientes con enfermedad por coronavirus nuevo (COVID - 19) . J Intern Med. 2020 ; 288 ( 1 ): 128 - 138.

12. Zietz M, Zucker J, Tatonetti NP. Associations between blood type and COVID-19 infection, intubation, and death. Nat Commun 2020 Nov 13;11(1):5761. doi: 10.1038/s41467-020-19623-x.

13. Hakan G, Elifcan Aladağ K, Haluk D, Çağlayan Merve Ayaz Ceylan, Yahya B, Ahmet Çağkan Inkaya, Salih A, Nilgün S, Ibrahim Celalettin H, Ömrüm U, Murat A, Osman IÖ, Serhat Ü. The effects of blood group types on the risk of COVID-19 infection and its clinical outcome. Turk J Med Sci . 2020 Jun 4;50(4):679-683. doi: 10.3906/sag-2005-395. Online ahead of print.

14. Yuqin W, Zhicai F, Peng L, Qizhi Y. Relationship between ABO blood group distribution and clinical characteristics in patients with COVID-19. Clin Chim Acta . 2020 Oct;509:220-223. doi: 10.1016/j.cca.2020.06.026. Epub 2020 Jun 17.

15. Nienhaus A, Hod Int J Environ Res Public Health. 2020 Jul; 17(13): 4881. doi: 10.3390/ijerph17134881

16. Skowronski. DM, Zou M, Clarke Q, et al. Influenza vaccine does not increase the risk of coronavirus or other non-influenza respiratory viruses: retrospective analysis from Canada, 2010-11 to 2016-17. Clin. Infect. Dis 2020; ciaa626.

17. Marin-Hernandez. D, Schwartz RE, Nixon DF. Epidemiological evidence for association between higher influenza vaccine uptake in the elderly and lower COVID-19 deaths in Italy. J Med Viril. 2020; 1-2. Epidemiological evidence for association between higher influenza vaccine uptake in the elderly and lower COVID-19 deaths in Italy.

18. The possible beneficial adjuvant effect of influenza vaccine to minimize the severity of COVID-19. Medical Hypothesis 2020; 140.

19. Santaolalla A, Beckmann K, Kibaru J, Josephs D, Van Hemelrijck M, Irshad S. Association Between Vitamin D and Novel SARSCoV-2 Respiratory Dysfunction - A Scoping Review of Current Evidence and Its Implication for COVID-19 Pandemic. Front Physiol. 2020 26(11). doi: 10.3389/fphys.2020.564387. PMID: 33324234; PMCID: PMC7726316. 
20. Kumar R, Rathi H, Haq A, Wimalawansa SJ, Sharma A. Putative roles of vitamin D in modulating immune response and immunopathology associated with COVID-19. Virus Res. 2021;292:198235. doi:10.1016/j.virusres.2020.198235

21. Blanco-Donoso, L.M., Garrosa, E., Moreno-Jiménez, J., Gálvez-Herrer, M., Moreno-Jiménez, B. 2020. Occupational psychosocial risks of health professionals in the face of the crisis produced by the COVID-19: From the identification of these risks to immediate action. International Journal of Nursing Studies Advances 2, 100003

22. German Federal Institute for Occupational Safety and Health (Bundesanstalt für Arbeitsschutz und

23. Arbeitsmedizin-BAuA). Empfehlungen der BAuA zum Einsatz von Schutzmasken im Zusammenhang mit

24. SARS-CoV-2. Available online: https://www.baua.de/DE/Themen/Arbeitsgestaltung-im-Betrieb/Biosto_e/FAQPSA/pdf/Schutzmasken.pdf?_blob=publicationFile\&v=12 (accessed on 27 April 2020).

25. 10. Weaver MD, Landrigan CP, Sullivan JP, et al. The association between resident physician work hour regulations and physician safety and health. The American Journal of Medicine 2020.

26. CDC COVID-19 Response Team. Characteristics of Health CarePersonnel with COVID-19 - United States, February 12-April9, 2020. MMWR Morb Mortal Wkly Rep. 2020;69:477--81,http://dx.doi.org/10.15585/mmwr.mm6915e6.

27. Kluytmans M, Buiting A, Pas S, Bentvelsen R, van den BijllaardtW, van Oudheusden A, et al. SARS-CoV-2 infection in 86 healthcare workers in two Dutch hospitals in March 2020. medRxiv.2020, http://dx.doi.org/10.1101/2020.03.23.20041913.7 